\title{
Low frequency acoustic and dielectric measurements on glasses
}

\author{
J. Classen, C. Enss, C. Bechinger ${ }^{1}$, G. Weiss ${ }^{2}$, and S. Hunklinger \\ Universităt Heidelberg, Institut für Angewandte Physik, \\ Albert-Ueberle-Str. 3-5, D-69120 Heidelberg, Germany
}

\begin{abstract}
The acoustic and dielectric properties of different glasses at audio frequencies and temperatures below $1 \mathrm{~K}$ have been investigated with the vibrating reed and a capacitance bridge technique. We found the temperature dependence of the absorption of vitreous silica (Suprasil W) to agree with the predictions of the tunneling model which is commonly used to explain the low temperature behaviour of amorphous materials. The variation of the sound velocity and of the dielectric constant, however, shows significant deviations from the expected behaviour which cannot be accounted for by a simple modification of the model. Instead, it seems to be necessary to introduce a temperature dependence of some relevant model parameters. Moreover, at very low temperatures $(T<0.1 \mathrm{~K})$ the sound velocity strongly depends on the excitation levels. The absence of this effect at higher temperatures proves that it can be ascribed to a nonlinear response of tunneling systems. Similar results were found in sound velocity measurements on a cover glass and on a superconducting metallic glass $\left(\mathrm{Pd}_{30} \mathrm{Zr}_{70}\right.$, $T_{\mathrm{c}}=2.6 \mathrm{~K}$ ), which indicates that these features are a general aspect of the dynamics of tunneling states in glasses. In contrast to the insulating glasses we found that in $\mathrm{Pd}_{30} \mathrm{Zr}_{70}$ also the internal friction is strain dependent.
\end{abstract}

Keywords: Tunneling states in glasses; Sound velocity; Internal friction; Dielectric constant.

\section{Introduction}

Numerous experiments of the last decades have shown that the low temperature properties of amorphous materials differ characteristically from those of pure crystals. The results of these thermal, acoustic and dielectric measurements [1] are to a large extent correctly described by the phenomenological tunneling model. According to this model the experimentally observed low energy excitations are formed by (for the first moment not specified) atoms or small groups of atoms which can carry out small configurational changes within the amorphous structure. In the simplest case they are described as particles in double well potentials. If the thermal energy $k_{B} T$ is much smaller than the barrier height $V$ between the wells, tunneling still allows transitions between the ground states leading to the formation of two level systems. These tunneling motions,

1 Present address: Universităt Konstanz, Fakultăt für Physik, Universitătsstr. 10, D-78464 Konstanz, Germany.

2 Present address: Universität Karlsruhe, Physikalisches Institut, Engesserstr. 7, D-76131 Karlsruhe, Germany. 
which do not occur in the well ordered structure of very pure crystals, determine the surprisingly universal properties of glasses at low temperatures.

Due to the structural disorder in glasses the local environment for different tunneling systems will vary. Hence the parameters which characterize the tunneling systems must have a very broad distribution. Simplifying but nonetheless rather successful assumptions concerning these distribution functions have been suggested in the so-called standard tunneling model $[2,3]$. They allow a prediction of the temperature and frequency dependence of acoustic and dielectric experiments, which are sensitive to the dynamics and the density of states of the tunneling systems. The measured quantities are the internal friction $Q^{-1}$ and the dielectric loss angle $\tan \delta$, as well as the relative change of sound velocity $\delta v / v=\left[\left(v(T)-v\left(T_{0}\right)\right] / v\left(T_{0}\right)\right.$ and of the dielectric constant $\delta \varepsilon / \varepsilon . T_{0}$ denotes an arbitrary reference temperature. For temperatures below $1 \mathrm{~K}$ the predictions of the tunneling model have been essentially confirmed by former experiments (for example $\delta v / v$ and $Q^{-1}$ of vitreous silica [4]). First indications for deviations from the expected behaviour resulted from dielectric measurements on the borosilicate glass BK7 [5] and motivated more detailed studies.

In this paper we present low frequency dielectric measurements and vibrating reed experiments on vitreous silica (Suprasil W), which show that also for this very pure structural glass the behaviour of $\delta v / v$ and $\delta \varepsilon / \varepsilon$ deviates from the prediction of the tunneling model. We find similar deviations in vibrating reed experiments on a cover glass [6] and on the superconducting metallic glass $\mathrm{Pd}_{30} \mathrm{Zr}_{70}$, which is expected to behave like an insulating glass far below its transition temperature $\left(T_{\mathrm{c}} \approx 2.6 \mathrm{~K}\right)$. As a possible solution to the observed discrepancies it is suggested that the density of states of the tunneling systems is effectively temperature dependent due to a strong coupling to thermal phonons. In addition, we discovered that below $100 \mathrm{mK}$ the sound velocity of all samples depends strongly on the deformation amplitude [7]. Only at particular amplitudes our results agree with those of former measurements [4]. Similar nonlinearities have been reported recently by Esquinazi et al. [8].

Before we discuss our experimental results in sections 4 and 5, we will give in section 2 a survey of the predictions of the tunneling model relevant to our measurements. Section 3 contains a brief description of the methods used in our experiments.

\section{The tunneling model}

The tunneling model is based on the assumption that in an amorphous solid for a small number of atoms or groups of atoms various equilibrium positions are accessible which have only a small difference in energy. In the simplest case these systems are described as particles in a double well potential consisting of two harmonic potentials (Fig. 1).

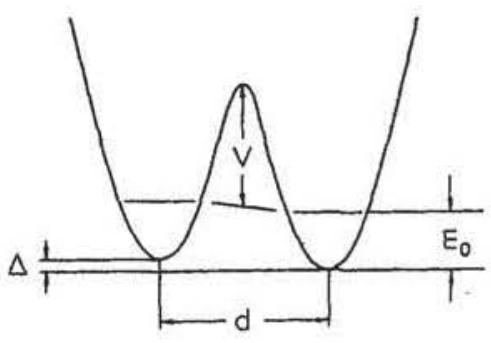

Fig. 1 Double well potential with barrier height $V$, asymmetry energy $\Delta$ and distance $d$. 
Due to local variation of the environment the minima of the wells may differ by an asymmetry energy $\Delta$. The two ground states in the wells are separated by the barrier height $V . m$ denotes the mass of the particle and $d$ the distance of the wells in configurational space.

At temperatures $T \ll V / k_{\mathrm{B}}$ the thermal energy is not sufficient for the particle to overcome the potential barrier, but transitions are still possible by tunneling processes. This tunneling motion results from the overlap of the ground state wavefunctions of the single wells and leads to a tunneling splitting $\Delta_{0}$ of the ground state energy $E_{0}$. Using the WKB method $\Delta_{0}$ can be estimated to

$$
\Delta_{0}=\frac{2 E_{0}}{\pi} e^{-\lambda}
$$

with the tunneling parameter

$$
\lambda \simeq \frac{d}{2 \hbar} \sqrt{2 m V} .
$$

The total energy splitting between the two states is

$$
E=\sqrt{\Delta^{2}+\Delta_{0}^{2}} .
$$

A further important postulate of the tunneling model concerns the distribution of the parameters $\Delta$ and $\lambda$ characterizing the tunneling systems. In a disordered structure these parameters will not have the same value for all systems but vary over a wide range. In the so-called standard tunneling model $\Delta$ and $\lambda$ are assumed to be independent of each other and uniformly distributed as

$$
P(\Delta, \lambda) \mathrm{d} \Delta \mathrm{d} \lambda=\breve{P} \mathrm{~d} \Delta \mathrm{d} \lambda .
$$

Although this assumption seems somewhat arbitrary it is essentially confirmed by results of many low temperature experiments $[1,4]$. It turns out, however, that the understanding of various measurements above $10 \mathrm{~K}$, for example of the temperature dependence of internal friction in vitreous silica, requires a modification of the distribution function [9]. But we want to point out that this modification does not imply any significant changes of the predictions of the properties below $1 \mathrm{~K}$ and hence will not be considered further in our discussion.

At this point we want to mention, that an equivalent description of the low temperature properties of glasses is given by the so-called KKI-model [10], which uses a more general form of the atomic potentials. In comparison to the standard tunneling model this leads to a slightly different distribution of the tunneling parameter. For the low temperature properties of the quantities discussed in this paper, however, the KKImodel yields the same predictions as the standard tunneling model [11]. Therefore we will not discuss the KKI-model in detail, but derive the dynamical properties of glasses in the framework of the standard tunneling model.

Tunneling systems couple to their environment by interaction with phonons and photons, in metals also with electrons. External elastic or electric fields produce changes of the asymmetry energy $\Delta$. A possible coupling to the tunneling splitting $\Delta_{0}$ 
is usually negligible compared to the fmodulation of $\Delta$ [12]. The variation $\delta \Delta$ for small perturbation should be proportional to the elastic deformation $e$ and the electric field $\vec{F}$, respectively:

$$
\delta \Delta=2 \gamma e \text { and } \delta \Delta=2 \vec{p} \cdot \vec{F}
$$

with the deformation potential $\gamma=(\partial \Delta / \partial e) / 2$ and the permanent electric dipole moment $\vec{p}$ of the tunneling systems. The quantities $\gamma$ and $e$ are tensors. Appropriate averages over their components will need to be taken since the orientation of the two level systems will vary from site to site [13]. In the case of the dipole moment only its component parallel to the applied field is relevant and therefore an averaged value $(\vec{p} \cdot \vec{F})^{2} /|\vec{F}|^{2}=p^{2} / 3$ will be used. Deformation potential and dipole moment are considered in this description - a further approximation of the standard tunneling model - as fixed quantities having the same value for all systems.

The equations of motion for the dynamics of the tunneling systems are the so-called Bloch equations. A general discussion of their solutions was given in [14]. For our measured quantities, in the acoustic case the relative change of sound velocity $\delta v / v$ and the internal friction $Q^{-1}$, in the dielectric case the relative change of the dielectric constant $\delta \varepsilon / \varepsilon$ and the dielectric loss angle $\tan \delta$ one finds in the low frequency limit, separated into relaxation and resonant contributions

$$
\begin{aligned}
& \left.\frac{\delta v}{v}\right|_{\text {rel }} \cdot \frac{-2 \varrho v^{2}}{\gamma^{2}}=\left.\frac{\delta \varepsilon}{\varepsilon}\right|_{\text {rel }} \cdot \frac{3 \varepsilon_{0} \varepsilon}{p^{2}}=\frac{1}{k_{\mathrm{B}} T}\left(\frac{\Delta}{E}\right)^{2} \operatorname{sech}^{2}\left(\frac{E}{2 k_{\mathrm{B}} T}\right) \frac{1}{1+(\omega \tau)^{2}} \\
& Q_{\text {rel }}^{-1} \cdot \frac{\varrho v^{2}}{\gamma^{2}}=\tan \delta_{\text {rel }} \cdot \frac{3 \varepsilon_{0} \varepsilon}{p^{2}}=\frac{1}{k_{\mathrm{B}} T}\left(\frac{\Delta}{E}\right)^{2} \operatorname{sech}^{2}\left(\frac{E}{2 k_{\mathrm{B}} T}\right) \frac{\omega \tau}{1+(\omega \tau)^{2}} \\
& \left.\frac{\delta v}{v}\right|_{\text {res }} \cdot \frac{\varrho v^{2}}{\gamma^{2}}=\left.\frac{\delta \varepsilon}{\varepsilon}\right|_{\text {res }} \cdot \frac{-3 \varepsilon_{0} \varepsilon}{2 p^{2}}=\frac{1}{E}\left(\frac{\Delta_{0}}{E}\right)^{2} \tanh \left(\frac{E}{2 k_{\mathrm{B}} T}\right) \\
& Q_{\text {res }}^{-1} \approx 0, \tan \delta_{\text {res }} \approx 0 .
\end{aligned}
$$

These equations are valid for an ensemble of identical tunneling systems after division by their number. $Q$ denotes the mass density and $\varepsilon_{0}$ the static dielectric constant.

In the case of the resonant interaction a quantum of the perturbation field - a phonon in the acoustic and a photon in the electric case - with energy $\hbar \omega$ is absorbed by a tunneling system with $E=\hbar \omega$, or, if the upper level is occupied, the emission of a further quantum is induced. In our case resonant absorption does not occur (Eq. 9), because for audio frequencies the resonant tunneling states $\left(E / k_{\mathrm{B}} \leq 1 \mu \mathrm{K}\right)$ are equally occupied.

Relaxation processes, in contrast, are caused by the modulation of the energy splitting $E$ due to the perturbation $\delta \Delta$. The occupation number difference between the two levels of an ensemble of identical tunneling systems does not correspond to thermal equilibrium any more, the systems relax with a particular time constant towards the new equilibrium. If this relaxation time $\tau$ is comparable to the period of perturbation ( $\omega \tau \approx 1$ ), absorption occurs. The relaxation time $\tau$ depends of course on the relaxation 
mechanism. Various low temperature experiments on glasses $[15,16]$ have shown that below $1 \mathrm{~K}$ the dominant relaxation mechanism in insulating glasses is the so-called one phonon or direct process with the rate [17]

$$
\tau_{\mathrm{d}}^{-1}=\frac{1}{2 \pi \varrho \hbar^{4}}\left(\frac{\gamma_{1}^{2}}{v_{\mathrm{l}}^{5}}+2 \frac{\gamma_{\mathrm{t}}^{2}}{v_{\mathrm{t}}^{5}}\right) E^{3}\left(\frac{\Delta_{0}}{E}\right)^{2} \operatorname{coth}\left(\frac{E}{2 k_{\mathrm{B}} T}\right) .
$$

The indices 1 and $t$ stand for longitudinal and transversal, respectively. Obviously, tunneling systems with fixed energy splitting $E$ may have a wide range of different relaxation rates depending on the ratio $\Delta_{0} / E$. The relaxation time of the fastest, i.e. symmetric, systems $\left(\Delta_{0} / E=1, \Delta=0\right)$ will be denoted in the following as $\tau_{\min }$.

In metallic glasses the interaction with conduction electrons is generally the most effective relaxation channel [18]. In the case of superconducting metallic glasses, however, this process freezes out below the transition temperature $T_{\mathrm{c}}$ due to the formation of Cooper pairs. In the limiting case $T \ll T_{c}$ one finds for tunneling systems which have a small energy splitting compared to the energy gap in the electronic spectrum $\left(E \ll 2 \Delta_{\mathrm{BCS}}(T)\right)$ the relaxation rate [19]

$$
\tau_{\mathrm{el}, \mathrm{s}}^{-1}=\frac{2 \pi}{\hbar} K k_{\mathrm{B}} T\left(\frac{\Delta_{0}}{E}\right)^{2} \frac{1}{1+\exp \left(\Delta_{\mathrm{BCS}}(T) / k_{\mathrm{B}} T\right)},
$$

where the dimensionless parameter $K$ contains an appropriate coupling constant and the electronic density of states at the Fermi level. Far below $T_{\mathrm{c}}$ relaxation via phonons will dominate again, and metallic glasses behave dynamically like dielectric glasses [20].

To obtain the contribution of all tunneling systems to our measured quantities Eqs. $6-9$ have to be integrated over $\Delta$ and $\lambda$, weighted with the distrbution function $P(\Delta, \lambda)$ (Eq. 4). One finds for the resonant contribution to the sound velocity and the dielectric constant [4]

$$
\left.\frac{1}{C_{\mathrm{a}}} \frac{\delta v}{v}\right|_{\text {res }}=-\left.\frac{1}{C_{\mathrm{d}}} \frac{\delta \varepsilon}{\varepsilon}\right|_{\text {res }}=\ln \left(\frac{T}{T_{0}}\right)
$$

where

$$
C_{\mathrm{a}}=\frac{\bar{P} \gamma^{2}}{\varrho v^{2}} \quad \text { and } \quad C_{\mathrm{d}}=\frac{2}{3} \frac{\bar{P} p^{2}}{\varepsilon_{0} \varepsilon}
$$

are material dependent constants for the acoustic and dielectric case, respectively.

Assuming that the relaxation takes place only via the direct process $\left(\tau^{-1}=\tau_{\mathrm{d}}^{-1}\right)$, in the limiting cases $\omega \tau_{\min } \ll 1$ and $\omega \tau_{\min } \gg 1$ analytical expressions can be derived for the relaxation contributions (Eqs. 6, 7). One finds for $\omega \tau_{\min } \ll 1$

$$
\begin{aligned}
& \frac{1}{C_{\mathrm{a}}} Q_{\mathrm{rel}}^{-1}=\frac{2}{C_{\mathrm{d}}} \tan \delta_{\mathrm{rel}}=\frac{\pi}{2} \\
& \left.\frac{1}{C_{\mathrm{a}}} \frac{\delta v}{v}\right|_{\mathrm{rel}}=-\left.\frac{1}{C_{\mathrm{d}}} \frac{\delta \varepsilon}{\varepsilon}\right|_{\mathrm{rel}}=-\frac{3}{2} \ln \left(\frac{T}{T_{0}}\right)
\end{aligned}
$$


and for $\omega \tau_{\min } \gg 1$

$$
\begin{aligned}
& \frac{1}{C_{\mathrm{a}}} Q_{\mathrm{rel}}^{-1}=\frac{2}{C_{\mathrm{d}}} \tan \delta_{\text {rel }} \propto \frac{T^{3}}{\omega} \\
& \left.\frac{\delta v}{v}\right|_{\mathrm{rel}} \simeq 0,\left.\frac{\delta \varepsilon}{\varepsilon}\right|_{\mathrm{rel}} \simeq 0 .
\end{aligned}
$$

The results for the absorption (Eqs. 14 and 16) can be understood quite easily. The main contribution to the absorption comes from systems with $E \simeq k_{\mathrm{B}} T$ and $\omega \tau \simeq 1$. At low temperatures and/or high frequencies the second condition cannot be fulfilled even by the fastest relaxing systems. Thus, according to the relation $\tau_{\min }^{-1} \propto E^{3}$, the absorption increases with $T^{3}$ as long as $\omega \tau_{\min } \gg 1$ holds. At sufficiently high temperatures or low frequencies, however, the damping is independent of temperature within a certain range, often referred to as "plateau", and independent of frequency. Fig. 2 shows the measured temperature dependence of the internal friction $Q^{-1}$ of vitreous silica at frequencies of 1.2 and $11.4 \mathrm{kHz}$. As expected, at low temperatures the damping increases with temperature and becomes independent of temperature above $1 \mathrm{~K}$ having the same value for both frequencies. At higher temperatures the potential barrier can be overcome by thermal activation leading to the rise of the internal friction above 3 or $4 \mathrm{~K}$. The decrease of $Q^{-1}$ above approximately $30 \mathrm{~K}$ can be attributed to a reduced number of defect systems with large barrier heights $V$ [9]. The contribution by thermally activated processes will not be discussed further, since we want to focus our attention to the temperature range below $1 \mathrm{~K}$.

Changes of the sound velocity and of the dielectric constant at low temperatures.are exclusively determined by resonant contributions (Eq. 12), whereas at higher'tem-

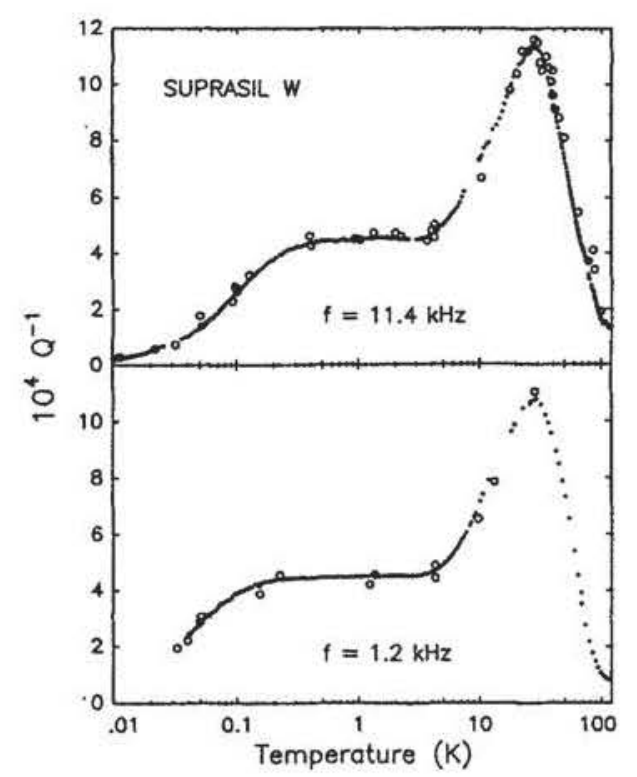

Fig. 2 Internal friction $Q^{-1}$ of Suprasil W for frequencies 11.4 and $1.2 \mathrm{kHz}$, respectively, as a function of temperature. Open symbols denote $Q^{-1}$ measurements by evaluation of complete resonance curves (see section 3 ). 
peratures relaxation processes according to Eq. 15 have to be taken into account. In this case the change of the two measured quantities is given by the sum of both processes:

$$
\left.\frac{1}{C_{\mathrm{a}}} \frac{\delta v}{v}\right|_{\text {tot }}=-\left.\frac{1}{C_{\mathrm{d}}} \frac{\delta \varepsilon}{\varepsilon}\right|_{\text {tot }}=-\frac{1}{2} \ln \left(\frac{T}{T_{0}}\right) \text { for } \omega \tau_{\min } \ll 1 .
$$

Using a logarithmic temperature scale for $\delta v / v$ and $\delta \varepsilon / \varepsilon$, the slope in the low temperature region and the slope at higher temperatures have a ratio of $2:-1$ in the case of sound velocity and $-2: 1$ in the case of the dielectric constant. Both regimes can be observed only in a relatively narrow frequency range. At too low frequencies the condition $\omega \tau \approx 1$ can be fulfilled even for the lowest temperatures experimentally accessible. In contrast, at $\mathrm{MHz}$-frequencies the relaxation contribution is negligible below $1 \mathrm{~K}$. Above $1 \mathrm{~K}$, however, the relaxation takes place not only via the direct process and therefore Eq. 15 is not valid any more. For our experiments, where temperatures down to approximately $10 \mathrm{mK}$ could be achieved, measuring frequencies of some $\mathrm{kHz}$ have been used so that the extremum of $\delta v / v$ and $\delta \varepsilon / \varepsilon$ occurred between 50 and $250 \mathrm{mK}$. This allowed a sensitive test of the predicted ratio of slopes.

\section{Experimental techniques}

Let us first discuss the experimental setup used for the dielectric experiments. In dielectric measurements on glasses at low temperature it is very difficult to determine the absolute value of the absorption because of its smallness and the low permissible excitation levels. To perform such measurements with sufficient resolution we have used a specially designed bridge circuit. It consists of an inductive voltage divider, a resistance decade, and a reference capacitor (Fig. $3 \mathrm{a}$ ). The essential difference from commercial capacitance bridges is that our reference capacitor, a sapphire disk $2 \mathrm{~mm}$ thick and $20 \mathrm{~mm}$ in diameter with evaporated gold electrodes, is integrated into the sample holder (Fig. $3 \mathrm{~b}$ ). At temperatures below $1 \mathrm{~K}$ the dielectric absorption of this material is
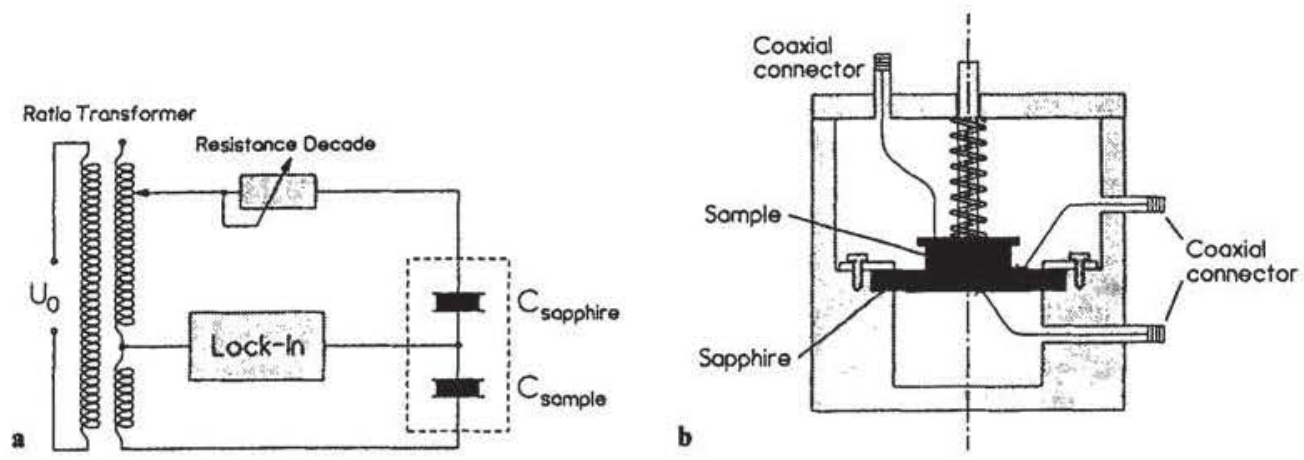

Fig. 3 The capacitance bridge. (a) Electrical circuit. The bridge is balanced by setting the voltage of the ratio transformer according to the ratio between the reference capacitance and the sample capacitance. The dielectric loss of the sample is compensated by the resistance decade. Bridge null is detected by a lock-in amplifier. The dashed line encloses components located in the cryostat. (b) Sample holder. The sample is mounted on a sapphire disk which simultaneously serves as reference capacitor. The surrounding sample holder is made of copper. 
negligible. One electrode of the reference capacitor also served as a sample electrode and was surrounded by a grounded guard ring. Connection between the room temperature components of the bridge and the sample holder was made with three coaxial cables. The glass samples were pressed onto the reference capacitor with a spring-loaded goldplated copper disk (Fig. 3 b). The sample holder was attached to the mixing chamber of a ${ }^{3} \mathrm{He} /{ }^{4} \mathrm{He}$ dilution refrigerator. Good thermal contact to the sample was ensured by the high thermal conductivity of the sapphire reference capacitor. The temperature was determined by a carbon resistance thermometer attached to the sample holder and by the magnetic susceptibility of a CMN thermometer located in the mixing chamber.

The sample was a disk of Suprasil W, $15 \mathrm{~mm}$ in diameter and $2 \mathrm{~mm}$ thick. Suprasil $\mathrm{W}$ is a very pure synthetic glass with a particularly low concentration of polar impurities such as $\mathrm{OH}^{-}$. The dielectric constant was determined from the setting of the ratio transformer and the value of the reference capacitance. Absorption was compensated by the resistance decade in the reference branch. The high symmetry and stability of this design resulted in a very low level of pick-up from outside sources and allowed to measure the relative change of the dielectric constant and the absorption with a resolution of about $10^{-7}$ and $10^{-6}$, respectively. The error in the absolute value of the absorption is mainly due to difficulties in precisely determining the residual absorption of the bridge circuit. This absorption is caused by losses in the cables and the ratio transformer. The error depends on the measuring frequency and was of the order of $10 \%$ for frequencies below $20 \mathrm{kHz}$.

The measurements of the relative change of sound velocity $\delta v / v$ and of the internal friction $Q^{-1}$ have been carried out with the vibrating reed technique [21]. A thin rectangular plate of the sample material is clamped at one end between two copper blocks, and the free end is driven electrostatically to forced vibrations [22]. Owing to the quadratic relation between acting force $F$ and voltage $U$ the reed vibrates with twice the driving frequency. Resonance occurs if one of the eigenmodes of the reed coincides with twice the frequency of the driving voltage. The lowest eigenfrequency of the plate, which has exclusively been used in our experiments, is given by $f_{\mathrm{r}}=0.1615\left(h / l^{2}\right) v_{\mathrm{Y}}$ with the thickness $h$ and the length $l$ of the sample and the sound velocity $v_{\mathrm{Y}}$, which is determined by Young's modulus $Y$ [23]. The absolute value of the sound velocity can be determined only coarsely with this equation, but since $\delta f_{\mathrm{r}} / f_{\mathrm{r}}=\delta v / v$ (at negligible thermal expansion) relative changes of the sound velocity can be measured by the shift of resonance frequency with an accuracy of the order of $10^{-6}$. A digital phase lock loop was used to keep the reed in resonance. It consisted of a phase-sensitive amplifier (lockin) and a synthesizer which was controlled by a personal computer (Fig. 4).

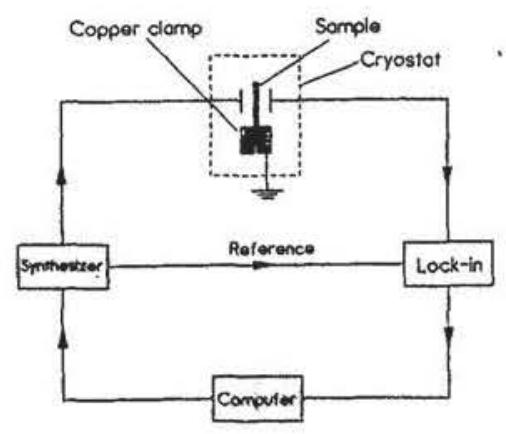

Fig. 4 Experimental setup for the vibrating reed experiments with digitally controlled phase-locked loop. The reed is electrostatically driven by the output of a synthesizer. The resulting vibration is detected by a lockin amplifier. 
The absolute value of the internal friction, $Q^{-1}=\Delta f / f_{\mathrm{r}}$, is determined by recording and evaluating resonance curves. $\Delta f$ denotes the width of the resonance at $A_{0} / \sqrt{2}$, where $A_{0}$ is the maximum amplitude. Using this method the error is typically a few percent. Keeping the reed in resonance it is possible to follow the variation of $Q^{-1}$ by simply measuring $A_{0}$, since $Q^{-1} \propto F_{0} A_{0}^{-1} f_{\mathrm{r}}^{-2}$, where $F_{0}$ denotes the amplitude of the driving force. By this, relative changes of the internal friction can be determined with errors of only a few per mil. While usually $F_{0}$ is fixed, with a digital circuit it is alternatively possible to measure $Q^{-1}$ by keeping the reed amplitude $A_{0}$ constant and recording the required variation of $F_{0}$. Suprasil $\mathrm{W}$ and the metallic glass $\mathrm{Pd}_{30} \mathrm{Zr}_{70}$ have been investigated at constant driving force, and both methods have been used for the cover glass sample [24].

\section{Experimental results}

\section{a) Suprasil $W$}

Figs. 5 and 6 show the results of the dielectric measurements of Suprasil W for different measuring frequencies. The dielectric losses (Fig. 5) are extremely small and thus difficult to measure. They have only been corrected by subtraction of the known residual absorption of the apparatus which is almost independent of temperature and frequency. The uncertainty of this correction yields a small uncertainty both of the absolute values and of the temperature dependence of $\tan \delta$. As expected from the tunneling model, we find $\tan \delta$ to increase strongly with increasing temperature at low temperatures and then to reach a temperature-independent region. Additionally, changing the frequencies from 2 to $20 \mathrm{kHz}$, the transition to the plateau regime is shifted from approximately 200 to $450 \mathrm{mK}$. The small difference between the plateau heights is within the experimental error of approximately $10 \%$ for the absolute value.

Fig. 6 shows the temperature dependence of the relative change of the dielectric constant. At low temperatures $\varepsilon$ decreases with increasing temperature neariy logarithmically, passes a minimum and increases at higher temperatures. The position of the mini-

Fig. 5 Dielectric loss angle $\tan \delta$ of Suprasil W for frequencies 2 and $20 \mathrm{kHz}$.

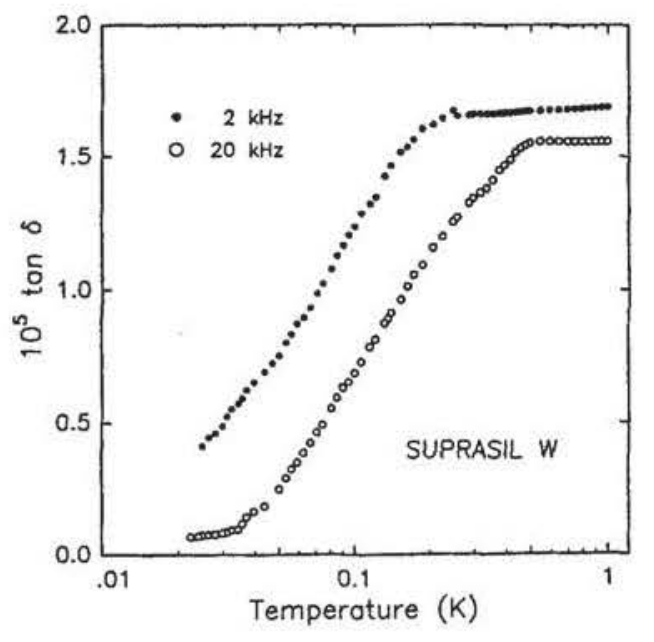




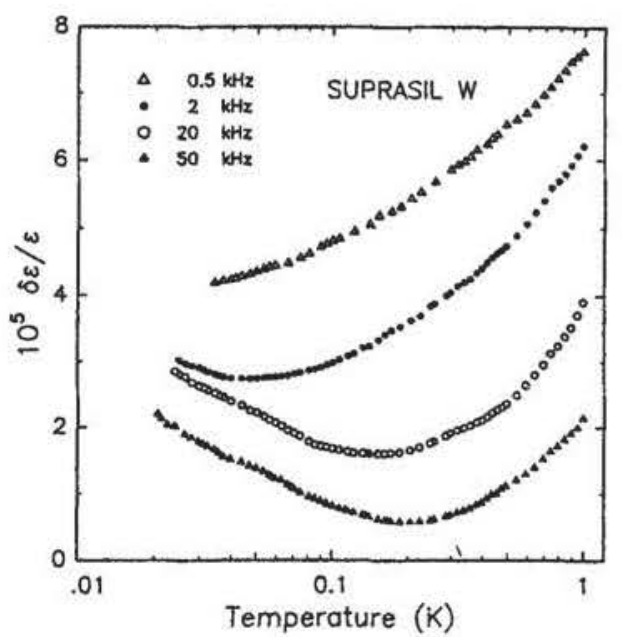

Fig. 6 Relative change with temperature of the dielectric constant $\delta \varepsilon / \varepsilon$ of Suprasil W for different frequencies.

mum shifts with increasing frequency to higher temperatures. These observations are in agreement with the tunneling model, but a more detailed analysis shows clearly deviations. First of all, the increase of $\delta \varepsilon / \varepsilon$ above the minimum is not really logarithmic but always bends to larger slopes. This might indicate that the relaxation behaviour of the tunneling systems is not exclusively determined by the direct process even at temperatures below $1 \mathrm{~K}$. Due to the bending of the curves it is difficult or even impossible to extract one well defined slope for the increase of $\delta \varepsilon / \varepsilon$. However, taking the change of $\varepsilon$ of the $2 \mathrm{kHz}$ measurement between 150 and $300 \mathrm{mK}$, where it varies nearly logarithmically, we find as a reasonable approximation the slope to be even slightly steeper than on the low temperature side, in obvious contradiction to the $-2: 1$ behaviour predicted by the tunneling model. Similar results for $\delta \varepsilon / \varepsilon$ have been obtained for the borosilicate glass BK 7 [5].

These results lead unavoidably to the question whether such strong deviations from the tunneling model can also be found in acoustic experiments, namely in measurements of the relative change of sound velocity, where - apart from the sign - the same qualitative picture is expected. Fig. 7 shows the temperature dependence of the sound velocity of Suprasil W measured in vibrating reed experiments. Again we find qualitatively the behaviour expected from the tunneling model: An increase of $\delta v / v$ at low temperatures and after passing a maximum, which shifts with increasing frequency to higher temperatures, a decrease. Unexpectedly, however, for both frequencies a strong dependence on the driving voltage occurs. While at higher temperatures the differences between the various curves are very small we find at very low temperatures a large decrease of the sound velocity with increasing driving voltage. Thus the steepness of the increase of $\delta v / v$ at low temperatures depends on the strain applied to the reed. For the $1.2 \mathrm{kHz}$ measurement a comparison of the slopes below and above the maximum is not possible since the region below the maximum is not wide enough. Analyzing the results of the $11.4 \mathrm{kHz}$ measurements, we find that the sound velocity does not vary strictly logarithmically on both sides of the maximum. Since the bending is rather small, however, a comparison of the slopes is possible. At the lowest driving voltage $(0.7 \mathrm{~V})$ the increase of $\delta v / v$ below the maximum is approximately as steep as the decrease at higher temperatures, i.e., the ratio of slopes is $1:-1$, similar to the 
Fig. 7 Relative change of the sound velocity $\delta v / v$ of Suprasil $\mathrm{W}$ for two frequencies. Different symbols denote different driving voltages. At low temperatures a strong amplitude dependence occurs.

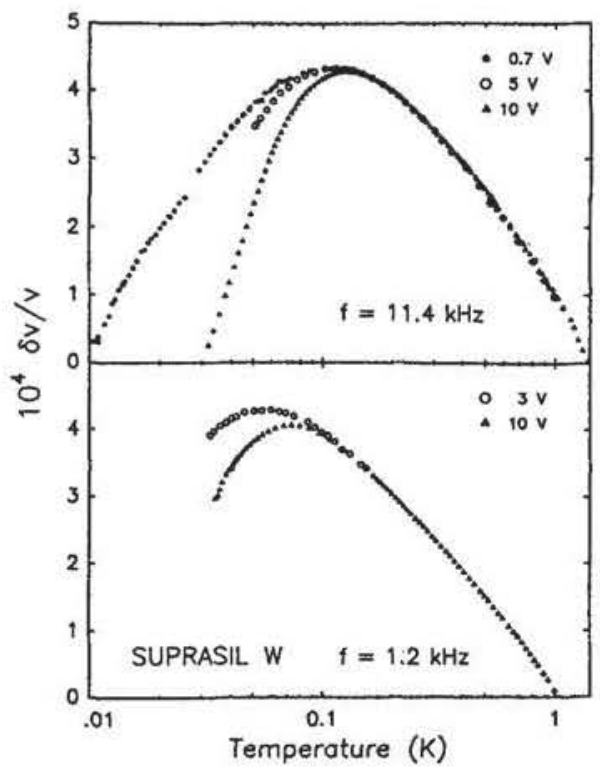

behaviour found for the dielectric constant. At a driving voltage of $10 \mathrm{~V}$ the increase of the sound velocity is much steeper yielding a ratio of slopes between 2: -1 and $3:-1$. In this case, since $e \propto U^{2}$, the generated deformations are more than two orders of magnitude larger than at $0.7 \mathrm{~V}$.

The nonlinear behaviour at low temperatures is also demonstrated by the shape of resonance curves. While at low driving voltages or strain amplitudes the resonance curves have the well known symmetric Lorentzian shape, they become more and more asymmetric with increasing voltage and shift to lower frequencies. Fig. 8 shows as an example three resonance curves where the amplitude of the reed has been multiplied with the cosine of the phase angle $\varphi$ between the driving force and the vibration. At $1.2 \mathrm{~V}$ the resonance curve is rather symmetric but going to $10 \mathrm{~V}$ we get a shift of $1.6 \mathrm{~Hz}$

Fig. 8 Resonance curves of the $11.4 \mathrm{kHz}$ Suprasil W reed at $32 \mathrm{mK}$ for three different driving voltages. The measured quantity is the amplitude of vibration multiplied with the cosine of the phase angle $\varphi$ between driving force and vibration. For clarity the data have been scaled up to similar magnitude.

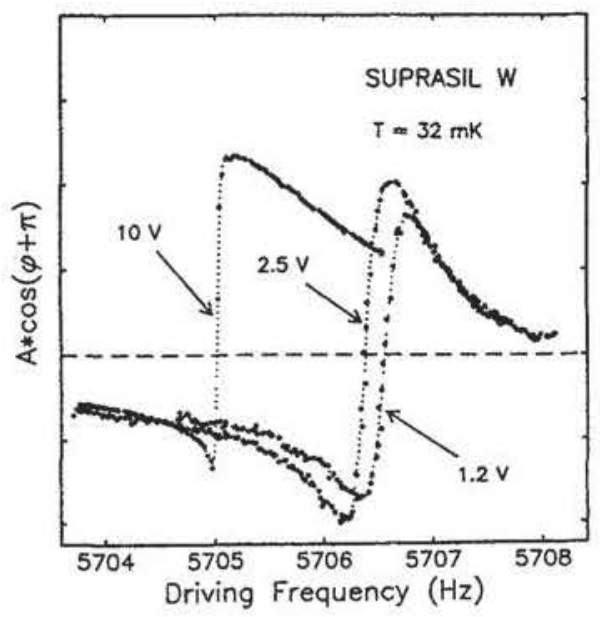


or $2.8 \times 10^{-4}$ corresponding to the decrease of $\delta v / v$ at $32 \mathrm{mK}$ in Fig. 7. The resonance curve has become very asymmetric and just below the resonance a steplike transition from negative to positive values of the signal occurs indicating strong nonlinear behaviour. The influence of the nonlinearities increases with decreasing temperature because the internal friction strongly decreases at lower temperatures (see Fig. 2). At fixed driving force the amplitude of vibration thus increases during cooling, and very small driving voltages are necessary to keep the nonlinearities negligible even at lowest temperatures. By carefully analyzing the resonance curves we know that in the case of the $0.7 \mathrm{~V}$ measurement we always stayed in the linear limit so that these results may be compared with the predictions of the tunneling model. What we find is a clear disagreement: The experimentally determined ratio of slopes is approximately $1:-1$ instead of $2:-1$ as discussed above. The internal friction, in contrast to the sound velocity, does not seem to show a definite strain dependence. In the case of very asymmetric resonance curves, however, even a rough determination of $Q^{-1}$ is difficult and thus a doubtless statement on strain dependence not possible. Although in our dielectric experiments no significant dependence on the amplitude of the electric field was observed, possibly due to the fact that only voltages less than $1 \mathrm{~V}$ have been applied, dielectric measurements on BK7 reported earlier by Frossati et al. [25] indicate that such a nonlinear behaviour is not an unique acoustic feature.

\section{b) Cover glass}

An interesting question is whether a similar behaviour as for Suprasil $\mathrm{W}$ can be found in other glasses, i.e., strong nonlinearities at low temperatures and, in the linear case, deviations from the tunneling model for the temperature dependence of $v$. In fact, the cover glass sample showed strong nonlinearities as well. Fig. 9 shows a resonance curve at $11 \mathrm{mK}$ as an text book-like example [26] where even hysteretic effects occur, i.e., the shape of the curve depends on the direction of the frequency sweep. A reduction of the driving voltage again yields symmetric resonance curves and thus linear

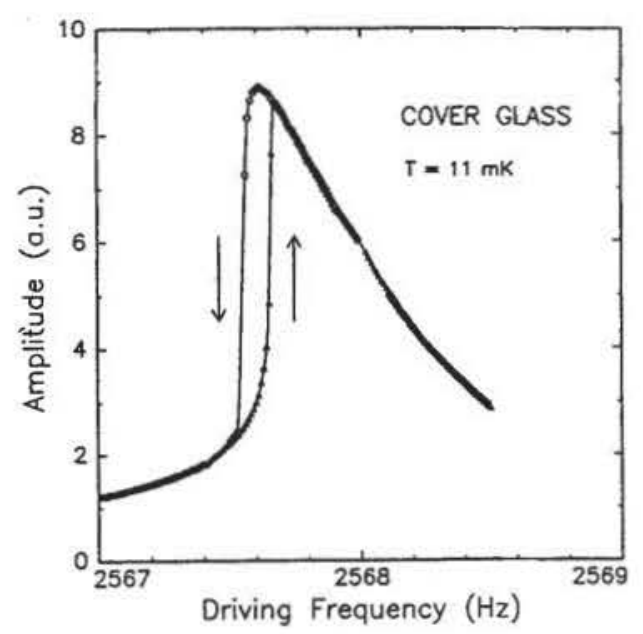

Fig. 9 Hysteretic resonance curve of the cover glass reed at high driving voltage. Arrows indicate the direction of the frequency sweep. 
Fig. 10 Relative change of the sound velocity $\delta v / v$ of a cover glass sample. Full symbols denote measurements where the vibrational amplitude of the reed was kept constant (the values $e=1 \times 10^{-7}$ and $e=2 \times 10^{-6}$ are estimates of the maximum strain amplitudes, which occurs at the clamping position). Open circles represent a measurement with constant driving voltage at an intermediate and temperature-dependent strain amplitude.

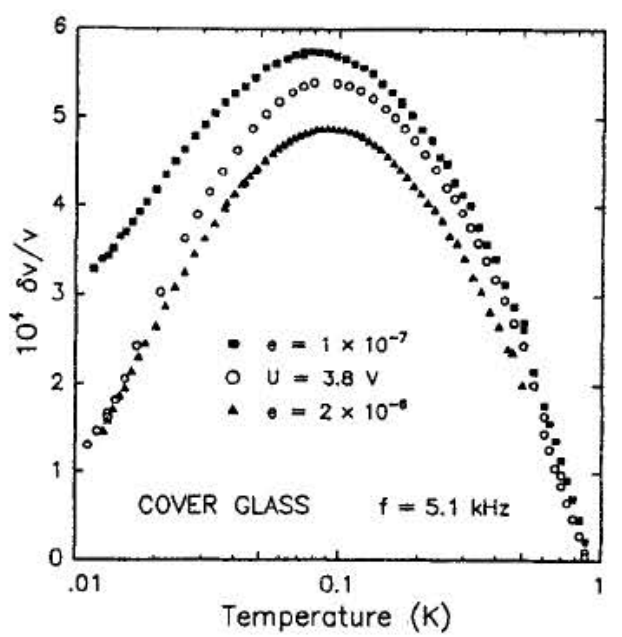

behaviour. The temperature dependence of the sound velocity is plotted in Fig. 10 . Since these measurements were performed with a digitally controlled phase-locked loop, the amplitude of the reed could be held constant although the internal friction changes strongly with temperature. The temperature dependence of $Q^{-1}$ looks very similar to that of Suprasil W (Fig. 2) and will therefore not be presented here. Two measurements were made with constant vibrational amplitudes, corresponding to maximum strain amplitudes of approximately $1 \times 10^{-7}$ and $2 \times 10^{-6}$ [27], and one measurement with constant driving voltage to demonstrate the differences between the two measuring methods. As for Suprasil W we find large differences between the various curves at very low temperatures and only small differences at higher temperatures. The temperature dependence is nowhere strictly logarithmic, but below $30 \mathrm{mK}$ and above $200 \mathrm{mK}$ the bending is small enough to allow a comparison of the slopes. In all cases the ratio of slopes below and above the maximum of the sound velocity deviates from the predicted 2: -1 behaviour. The smallest deviation shows the curve measured with fixed driving voltage (filled dots), whereas with constant vibrational amplitude of $10^{-7}$ (open circles) the increase at low temperatures is even flatter than the decrease above the maximum.

\section{c) $P d_{30} Z r_{70}$}

As a third material we have investigated a vibrating reed sample of the superconducting metallic glass $\mathrm{Pd}_{30} \mathrm{Zr}_{70}$. Figs. 11 and 12 show internal friction and relative change of sound velocity, respectively, for different driving voltages. All the $\mathrm{Pd}_{30} \mathrm{Zr}_{70}$ measurements were performed with constant driving voltage. Some features distinguished from our previous results are remarkable. First of all, the internal friction shows an unambiguous strain dependence. Secondly, at high voltages both measured quantities show a flattening at low temperatures, beginning below $40 \mathrm{mK}$ at $10 \mathrm{~V}$, and below $80 \mathrm{mK}$ at $20 \mathrm{~V}$. Extrapolating the thermal conductivity data of $\mathrm{Pd}_{30} \mathrm{Zr}_{70}$ [28] to lower temperatures, it can be easily estimated that the heat produced in the reed by the vibration, which is at $20 \mathrm{~V}$ of the order of some $10^{-10} \mathrm{~W}$, cannot be transported fast enough 

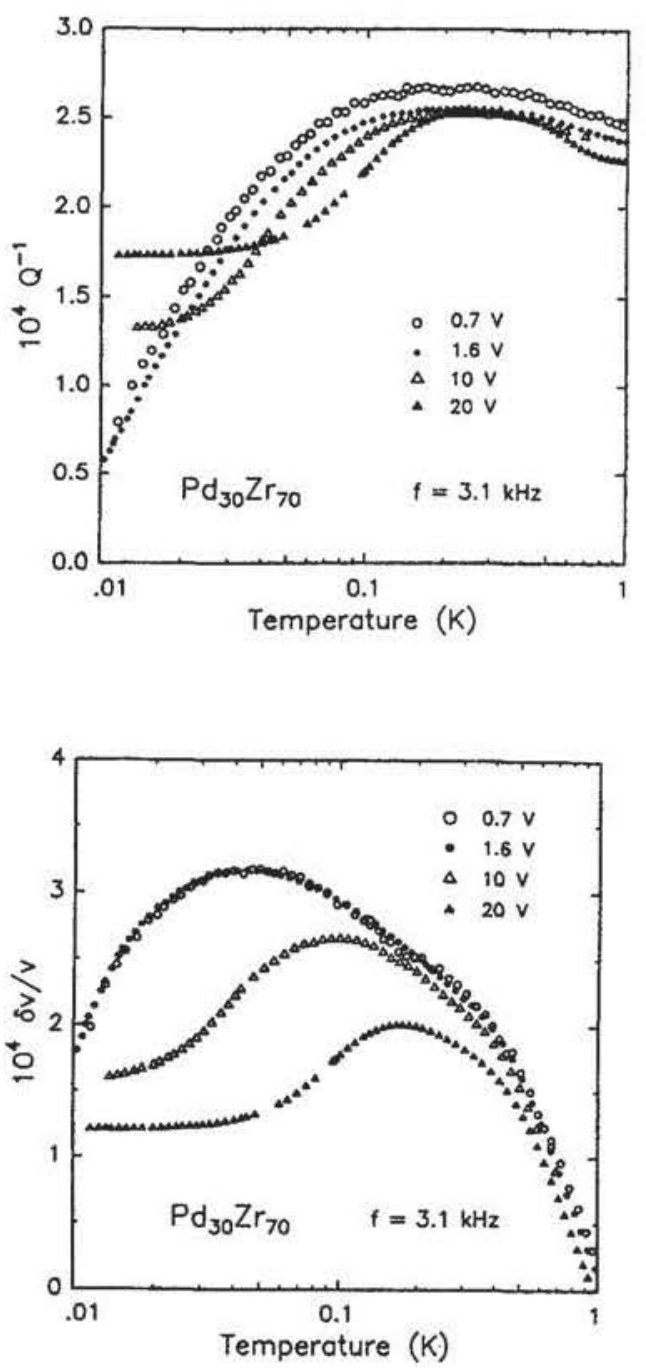

Fig. 11 Internal friction $Q^{-1}$ of $\mathrm{Pd}_{30} \mathrm{Zr}_{70}$ at $3.1 \mathrm{kHz}$ in the superconducting state for different driving voltages. Selfheating occurs below $30 \mathrm{mK}$ and $70 \mathrm{mK}$ for the measurements at $10 \mathrm{~V}$ and $20 \mathrm{~V}$, respectively, leading to a flattening of the data.

Fig. 12 Relative change of the sound velocity $\delta v / v$ of the $\mathrm{Pd}_{30} \mathrm{Zr}_{70}$ reed for different driving voltages. The high amplitude curves show the same flattening due to selfheating as the corresponding $Q^{-1}$ data of Fig. 11 .

through the cross-section of the sample and thus leads to selfheating. Similar selfheating effects did not occur in the data presented above mainly due to the fact that the dielectric glasses, in contrast to the metallic glass sample, were covered with a thin layer of gold. Although three magnitudes thinner, this metallic layer has a higher thermal conductivity than the dielectric material at very low temperatures. The second reason are the particularly large deformations up to approximately $10^{-5}$ which are applied to the PdZr-reed.

The internal friction measurements (Fig. 11) show a continuous reduction of $Q^{-1}$ with increasing driving voltage. Between 200 and $400 \mathrm{mK}$ the differences between all curves are quite small and the internal friction is nearly temperature independent (plateau region). The weak temperature dependence of $Q^{-1}$ above $400 \mathrm{mK}$ can be ascribed to the onset of the interaction between tunneling systems and conduction electrons. In this temperature range the condition $T \ll T_{\mathrm{c}}$ is no longer fulfilled and the conduction electrons might noticeably enhance the relaxation rates of the tunneling 
systems. A further indication of such an additional relaxation mechanism is the increased steepness in the change of sound velocity above $400 \mathrm{mK}$ (Fig. 12). These remarkable effects had been observed and discussed earlier [29-32] and will not be emphasized further here. A new observation of the present investigation is an amplitude dependence of $Q^{-1}$ above $400 \mathrm{mK}$ which cannot be explained along the same line.

The strain dependence below $200 \mathrm{mK}$ might be qualitatively understood in the framework of the theory of nonlinear relaxation absorption [33 - 35]. It can be estimated that in this temperature range the change of asymmetry energy $\delta \Delta=2 \gamma e_{0}$ (Eq. 5 with a typical value of $\gamma \approx 1 \mathrm{eV}$ ) can exceed the thermal energy $k_{\mathrm{B}} T$. Since the density of states of excited phonons decreases above $k_{\mathrm{B}} T$ the relaxation probability of these two level systems is reduced. This finally leads to smaller values of the internal friction. Since the ratio $2 \gamma e_{0} / k_{\mathrm{B}} T$ increases with decreasing temperature, it is plausible that the differences between the various curves become larger at lower temperatures until the effect is covered by selfheating. It should be mentioned, however, that the difference of approximately $10 \%$ (which is slightly larger than the error bars) between the measurements at low driving voltages $(0.7$ and $1.6 \mathrm{~V})$ cannot be explained in this way since for these curves in the whole temperature range the condition $2 \gamma e_{0} \varangle k_{\mathrm{B}} T$ was fulfilled and the symmetric shape of resonance curves always indicated linear behaviour. Together with the strain dependence between 0.4 and $1 \mathrm{~K}$ this problem remains unsolved.

The measurements of the relative change of sound velocity are drastically influenced by the choice of the driving voltage. While the differences between the curves at 0.7 and $1.6 \mathrm{~V}$ are very small, we see for example a dramatic shift of the maximum of $\delta v / v$ from 45 to $95 \mathrm{mK}$ at $10 \mathrm{~V}$ and $180 \mathrm{mK}$ at $20 \mathrm{~V}$. The low temperature behaviour at high amplitudes is determined both by nonlinearities and by selfheating. The nonlinearities lead to a reduction of the sound velocity whereas the selfheating causes the flattening of the curves thus prohibiting a further decrease of the sound velocity to lower temperatures. For a comparison of the experimental data with the predictions of the tunneling model we have again to pay attention to the measurements in the linear limit, i.e., to the 0.7 or $1.6 \mathrm{~V}$ curves. Evaluating the slopes of the curves below and above the maximum (again the slope is not a well defined quantity since there is always a small bending, but below $15 \mathrm{mK}$ and between 150 and $300 \mathrm{mK}$ the data points nearly follow a straight line), we find their ratio to be approximately $3:-1$ again in disagreement with the tunneling model. Hence in contrast to the results for insulating glasses, the increase of $v$ at low temperatures is too steep compared to the decrease above the maximum. In the following section, where we will briefly summarize our observations, we will discuss this point and our further main results.

\section{Discussion}

According to the tunneling model the absorption behaviour of glasses at audio frequencies is exclusively determined by relaxation of tunneling systems and not by resonant processes. Hence measurements of $\tan \delta$ or $Q^{-1}$ allow a test of the theoretical ideas concerning the relaxation mechanisms. In our experiments (Figs. 2, 5, 11) both the dielectric and the acoustic losses essentially show the temperature dependence expected from the tunneling model thus indicating that the relaxation processes seem to be quite well understood. One remaining discrepancy should be mentioned, however: We always find that the initial rise of the absorption at low temperatures depends more 
weakly on temperature than $\propto T^{3}$ (Eq. 16). This is principally not surprising because there are always background losses of unknown magnitude - in vibrating reed experiments for instance mainly due to clamping of the sample - which can by far exceed the intrinsic losses at very low temperatures. If we subtract a constant background from our data (for example $1.8 \times 10^{-5}$ in Fig. 2) we get a better but not yet satisfactory agreement with theory. Since we do not know much about the mechanisms leading to the clamping losses we cannot decide whether they possibly have themselves a slight temperature dependence or, alternatively, the intrinsic losses do not show the $T^{3}$ dependence expected from theory. Since the clamping losses might be caused by defects on the surface of the Cu-clamp they may even have a behaviour similar to that of metallic glasses. In any case, the background losses should always be small enough to be negligible at higher temperatures. In this region theory and experiment agree very well.

Using Eq. 14 we can estimate the values of the constants $C_{\mathrm{a}}$ and $C_{\mathrm{d}}$ for Suprasil W from the heights of the absorption plateaus in Figs. 2 and 5, respectively. These constants can also be determined from the steepness of the decrease of $\delta v / v$ above the maximum (Figs. 7, 10, 12) and the increase of $\delta \varepsilon / \varepsilon$ above the minimum (Fig. 6) using Eq. 18. A survey of the different values of $C_{\mathrm{a}}$ and $C_{\mathrm{d}}$ is given in Table 1 . The relatively large errors of $C_{\mathrm{a}}$ and $C_{\mathrm{d}}$ derived from the sound velocity and the dielectric constant measurements do not mainly result from the scatter of the data points, but from their curvature which principally prevents a more accurate determination. While the $C_{\mathrm{d}}$ values agree within the error limits, the $C_{\mathrm{a}}$ values of the acoustic experiments differ significantly. Similar discrepancies have already been reported earlier [4]. It is interesting to note that in our experiments these deviations can be correlated with the ratio of slopes of $\delta v / v$ below and above the maximum. As discussed in the previous section, an estimation of these ratios yields approximately $1:-1$ for Suprasil W, $1:-1.5$ for the cover glass and 3:-1 for $\mathrm{Pd}_{30} \mathrm{Zr}_{70}$ where we always regard the limiting case of linear behaviour.

With the argument that the relaxation contribution to the temperature dependence of the sound velocity is described correctly by theory as the internal friction data suggest, we come to the conclusion - if we do not want to give up the whole theory that the resonant contribution, or better, its magnitude relative to the relaxation part, mainly causes the deviations from the tunneling model. In fact, with a simple but arbitrary correction of the resonant contribution, we find a consistent description of the experimental situation: If the resonant contribution to the sound velocity were still logarithmic in $T$, but had a modified prefactor - for Suprasil W reduced by $20 \%$, for the cover glass by $40 \%$, and for $\mathrm{Pd}_{30} \mathrm{Zr}_{70}$ enlarged by $10 \%$ - the ratios of slopes

Table 1 Values $C_{\mathrm{a}}=\vec{P} \gamma^{2} / \varrho v^{2}$ and $C_{\mathrm{d}}=2 \vec{P} p^{2} / 3 \varepsilon_{0} \varepsilon$ in units of $10^{-4}$ determined from the plateau region of the acoustic $\left(Q^{-1}\right)$ or dielectric $(\tan \delta)$ absorption (Eq. 14) and from temperature variation of the sound velocity $\delta v / v$ above the maximum or dielectric constant $\delta \varepsilon / \varepsilon$ above the minimum (Eq. 18).

\begin{tabular}{llll}
\hline Experiment & Suprasil W & Cover glass & $\mathrm{Pd}_{30} \mathrm{Zr}_{70}$ \\
\hline$Q^{-1}$ & $2.8( \pm 0.15)$ & $3.1( \pm 0.15)$ & $1.65( \pm 0.1)$ \\
$\tan \delta$ & $0.205( \pm 0.015)$ & - & - \\
$\delta v / v$ & $3.7( \pm 0.4)$ & $5.3( \pm 0.6)$ & $1.31( \pm 0.15)$ \\
$\delta \varepsilon / \varepsilon$ & $0.22( \pm 0.05)$ & - & - \\
\hline
\end{tabular}


would be close to those experimentally observed. At the same time, the discrepancies between the differing $C_{\mathrm{a}}$ values, determined from the $Q^{-1}$ plateau and from $\delta v / v$ above the maximum, disappear since in this case the slopes of the logarithmic decrease of $\delta v / v$ above the maximum are expected to have the values $(0.8-1.5) C_{\mathrm{a}}=-0.7 C_{\mathrm{a}}$ (Suprasil W), $(0.6-1.5) C_{\mathrm{a}}=-0.9 C_{\mathrm{a}}$ (cover glass) and $(1.1-1.5) C_{\mathrm{a}}=-0.4 C_{\mathrm{a}}$ $\left(\mathrm{Pd}_{30} \mathrm{Zr}_{70}\right)$ instead of $-0.5 C_{\mathrm{a}}$ (Eq. 18). Thus we get from $\delta v / v \quad C_{\mathrm{a}}=2.65$ $( \pm 0.3) \times 10^{-4}$ (Suprasil W), $C_{\mathrm{a}}=2.9( \pm 0.4) \times 10^{-4}$ (cover glass) and $C_{\mathrm{a}}=1.64$ $( \pm 0.2) \times 10^{-4}\left(\mathrm{Pd}_{30} \mathrm{Zr}_{70}\right)$ in excellent agreement with the values determined from the plateau heights. A corresponding correction for the dielectric value $C_{\mathrm{d}}$, however, would yield $C_{\mathrm{d}}=1.57( \pm 0.4) \times 10^{-5}$ leading to a worse agreement than without correction but still within the error limits.

Following this line of description further, we tried to fit numerically our data over a wider range of temperatures. It was however not possible, particularly in the range $\omega \tau_{\min } \simeq 1$, to get satisfactory results for both internal friction and change of sound velocity with a single set of parameters. On the other hand, similar corrections to the predictions of the tunneling model had been introduced earlier to improve the agreement between internal friction and the resonant contribution to the sound velocity [36-38]. There, instead of Eq. 4, more sophisticated distribution functions had been used. The main feature of all these corrections is to modify the relative weight of "slow" and "fast" tunneling systems according to their ratio $\Delta_{0} / E$, irrespective however of the value of $E$. In our opinion, these procedures have two main problems. First, an additional parameter is created to improve only the phenomenological predictions. Without justification by a more microscopic theory this is at least unsatisfactory. Secondly, modifications or restriction of a distribution function with $\Delta_{0} / E$ as variable is not meaningful as long as $\Delta_{0}$ and $\Delta$ (or corresponding variables) are regarded as independently varying parameters.

To date there is no good solution to our stated discrepancies between the predictions of the tunneling model and the observed temperature dependence of the sound velocity. To emphasize this we show in Fig. 13 our sound velocity measurements on Suprasil W at $11.4 \mathrm{kHz}$ together with earlier data at lower frequencies [4] and more recent results from a torsional pendulum experiment at $66 \mathrm{kHz}$ [39]. The temperature variation of

Fig. 13 Comparison of different sound velocity measurements on Suprasil $\mathrm{W}$ in the $\mathrm{kHz}$ regime. The filled triangles show our $11.4 \mathrm{kHz}$ measurement in the case of small values of strain. Data at $66 \mathrm{kHz}$ are from [39] and data at $484 \mathrm{~Hz}$ and $3170 \mathrm{~Hz}$ from [4]. Lines are only a guide for the eye.

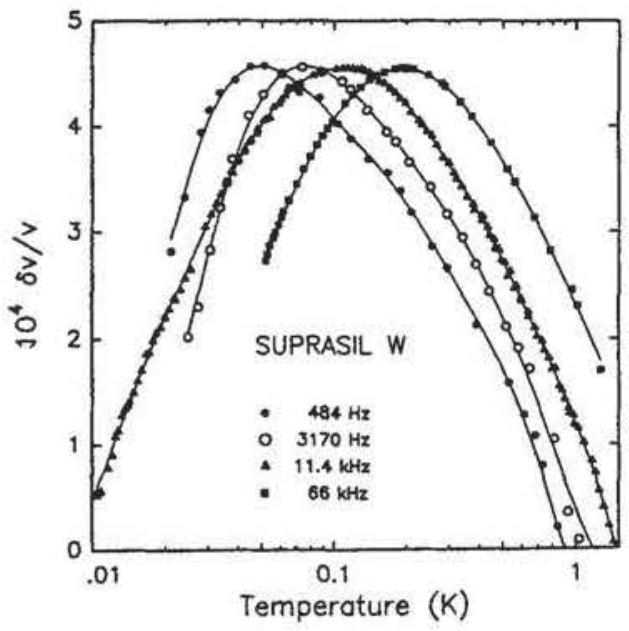


$\delta v / v$ above the maximum, which shifts according to the condition $\omega \tau_{\min }=1$, is in quantitative agreement for all frequencies. Below the maximum, the $66 \mathrm{kHz}$ experiment clearly supports our result in showing similar $1:-1$ behaviour of the slopes. The other two sets of data agree very well with our experiments at higher and constant driving voltages (see Fig. 7). We conclude therefore that in those experiments nonlinearities were present, which had not been discovered and correctly considered. Apparently, experiments at truly constant and very small amplitudes are indispensable for sensible tests of the predictions of the tunneling model.

Without going into further details we like to point out that within a linear response theory, applied to calculate the susceptibility of tunneling systems with asymmetric potentials, the above sketched predictions are difficult to change. As long as the tunneling systems are regarded as isolated and weakly interacting defects their relative contribution to resonant and relaxation processes only depends on the factor $\Delta_{0} / E$. And this factor is taken as a fixed quantity for each individual tunneling system. In a very recent investigation [40] it is shown, however, that strong coupling to the phonon bath may considerably reduce the phase coherence time of the tunneling systems and instead of $\Delta_{0}$ the energy $\Delta_{\text {res }}=\sqrt{\Delta_{0}^{2}-\Gamma_{2}^{2}}$ enters the expressions which describe the resonant interaction of tunneling systems with external fields. It is further shown that the inverse phase coherence time $\Gamma_{2}$ varies strongly with temperature, e.g. $\Gamma_{2} \propto T^{5}$ for a two phonon process. As a result, resonant effects will be weakened and additional relaxation contributions appear. Similar effects have been discussed for metallic glasses where short coherence times and renormalization occur due to a strong interaction of the tunneling systems with conduction electrons [41]. In terms of the phenomenological theory summarized in Chapter 2 these effects may be taken into account by introducing a temperature-dependent distribution function $P\left(\Delta, \Delta_{\text {res }}(T)\right)$. In the case of metallic glasses this concept seems to be able to solve some discrepancies between experiments and predictions of the tunneling model [29-32, 42]. A conclusive prediction for insulating glasses is however not possible yet.

In a final remark we like to come back to our observation of strong nonlinearities in the temperature dependence of the sound velocity. As we have shown these nonlinearities manifest themselves in a shift to lower frequencies and a strong distortion of the resonance curves. At this point one has to ask whether these effects are caused by tunneling systems or by nonlinearities of the amorphous structure itself. In Fig. 14 we have plotted the shift of the resonance frequency of Suprasil W as a function of the applied driving voltage at three different temperatures. Clearly, the frequency shift occurs only at lowest temperatures. It is absent in the temperature region of the absorption plateau, as stated above, but also at $113 \mathrm{~K}$ where $Q^{-1}$ is very small resulting in relatively large vibration amplitudes at a given excitation voltage. This demonstrates that the nonlinear behaviour is indeed a feature of the tunneling systems and not of the amorphous matrix or the experimental technique itself. Moreover, since the effect occurs mainly at temperatures below the maximum in $\delta v / v$, i.e. in the region $\omega \tau_{\min } \gg 1$, it must be caused by resonantly interacting tunneling systems.

In a recent paper this effect has been treated theoretically [43]. Introducing explicitly into Eq. (3) the modulation of $\Delta$ by the sound wave like $E^{2}=\Delta_{0}^{2}+\left(\Delta+2 \gamma e_{0}\right.$ $\sin (\omega t)^{2}$, it was argued that at sufficiently high strain amplitudes $e_{0}$ the time averaged energy splitting of a tunneling system is enlarged. Consequently, since $\omega \tau \gg 1$, the occupation numbers of the upper level would decrease as if the sample were at a lower temperature. This finally should lead to a reduced sound velocity and a steeper than a $\log T$ temperature dependence of $\delta v / v$. A numerical treatment [8], introducing a 
Fig. 14 Relative shift of the resonance frequency $\delta f_{\mathrm{r}} / f_{\mathrm{r}}$ of the $1.2 \mathrm{kHz}$ Suprasil $\mathrm{W}$ reed as a function of the driving voltage $U$ for different temperatures. Values of the internal friction at $40 \mathrm{mK}, 153 \mathrm{mK}$, and $113 \mathrm{~K}$ are $2.3 \times 10^{-4}, 4.2 \times 10^{-4}$, and $0.96 \times 10^{-4}$, respectively.

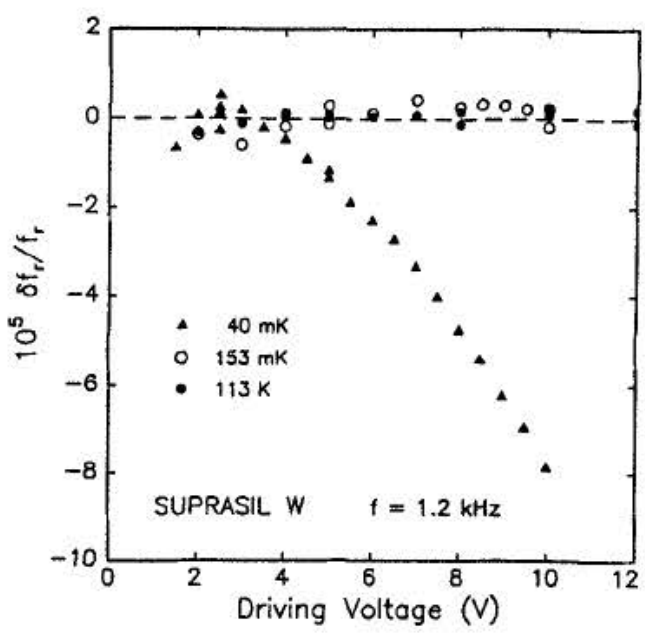

similar strain dependence in energy splitting and thus in occupation numbers, indeed showed satisfactory agreement with experiment. In [43] analytical expressions are derived and at very low temperatures and high strain amplitudes $\left(2 \gamma e_{0} \gg k_{\mathrm{B}} T\right)$ a behaviour proportional to $\left(T / e_{0}\right)^{2}$ is predicted. So far, this behaviour has not been observed. However, our experiments are not a quantitative test for the quadratic $T$ dependence since most of our measurements are done at constant driving voltage rather than constant deformation and, moreover, we hardly entered the region where $2 \gamma e_{0}$ is larger than thermal energy. Thus a conclusive statement on the predicted $\left(T / e_{0}\right)^{2}$ behaviour cannot be made at this point.

\section{Summary}

We have presented a series of experiments on the elastic and dielectric behaviour of various glasses in the $\mathrm{mK}$ temperature range. The overall temperature dependence up to a few $\mathrm{K}$ of absorption and sound velocity or dielectric constant is qualitatively well described by the tunneling model, where the susceptibility of two-well tunneling systems is treated in a linear response theory. Some of our measurements, however, namely the temperature dependence of the real part of the elastic or dielectric response, show considerable quantitative deviations from these predictions. In agreement with theory the sound velocity first increases logarithmically with temperature due to resonant processes. With rising temperature relaxation processes become important leading to a maximum, which occurs around $50 \mathrm{mK}$ at a measuring frequency of $1 \mathrm{kHz}$, and a subsequent decrease, again proportional to $\log T$, of the sound velocity. From theory a well defined ratio of $2:-1$ of the $\log T$ slopes of the two regimes is predicted. In our experiments, however, we find e.g. a ratio of 1: -1 for vitreous silica (Suprasil W). Corresponding discrepancies occur in the temperature dependence of the dielectric constant. Similar observations in other glasses demonstrate the general character of this problem. These deviations may be explained by a suppression of resonant processes due to a strong coupling of the tunneling systems to thermal phonons. Additionally, we have observed strong nonlinearities in the temperature dependence of the sound velocity, which also call for further theoretical consideration. 
We thank D. Parshin and M. v. Schickfus for fruitful discussions and P. Esquinazi for valuable comments and communicating to us his various experimental results.

\section{References}

[1] See for a review: W.A. Phillips (ed.), Amorphous Solids - Low Temperature Properties, Topics in Current Physics 24, Springer, Berlin Heidelberg New York 1981

[2] W.A. Phillips, J. Low Temp. Phys. 7 (1972) 351

[3] P.W. Anderson, B.I. Halperin, C.M. Varma, Philos. Mag. 25 (1972) 1

[4] A.K. Raychaudhuri, S. Hunklinger, Z. Phys. B57 (1984) 113

[5] C. Enss, C. Bechinger, M. v. Schickfus, Phonons 89, eds. S. Hunklinger, W. Ludwig, G. Weiss, World Scientific, Singapore 1989, 474

[6] This sample was a commercial glass slide as it is used for microscopy purposes. The exact composition of this silica-based glass is not known

[7] J. Classen, Diploma thesis, Universităt Heidelberg (1991), unpublished

[8] P. Esquinazi, R. König, F. Pobell, Z. f. Phys. B87 (1992) 305

[9] D. Tielbürger, R. Merz, R. Ehrenfels, S. Hunklinger, Phys. Rev. B 45 (1992) 2750.

[10] F.N. Ignatiev, V. G. Karpov, M.I. Klinger, J. Non-Cryst. Sol. 55 (1983) 307

[11] U. Buchenau, Yu.M. Galperin, V.L. Gurevich, D.A. Parshin, M.A. Ramos, H.R. Schober, Phys. Rev. B46 (1992) 2798; D.A. Parshin, Phys. Rev. B 49 (1994) 9400

[12] W.A. Phillips, in Ref. [1], p. 1

[13] B.I. Halperin, Ann. N.Y. Acad. Sci. 279 (1976) 173

[14] S. Hunklinger, W. Arnold, Physical Acoustics 12, eds. R. N. Thurston, W.P. Mason, Academic Press, New York 1976, 155

[15] S. Hunklinger, A.K. Raychaudhuri, Progress in Low Temp. Phys. Vol. IX, ed. D.F. Brewer, Elsevier, Amsterdam 1986, 265

[16] B. Golding, J.E. Graebner, in Ref. [1], p. 107

[17] J. Jäckle, Z. Phys. 257 (1972) 212

[18] J.L. Black, Glassy Metals 1, Topics in Applied Physics 46, eds. H. J. Guntherodt, H. Beck, Springer, Berlin Heidelberg New York 1981, 167

[19] J.L. Black, P. Fulde, Phys. Rev. Lett. 43 (1979) 453

[20] G. Weiss, B. Golding, Phys. Rev. Lett. 60 (1988) 2547

[21] B.S. Berry, W.C. Pritchet, IBM J. Res. Develop. 19 (1975) 334

[22] Insulating glasses must be covered with a thin metallic layer to get an electric conductivity. By sputtering we have deposited on our samples a $30-50 \mathrm{~nm}$ microcrystalline gold layer thin enough to exclude a noticeable influence on our measured quantities

[23] $v_{Y}$ is related to the longitudinal sound velocity $v_{l}$ by Poisson's ratio $\sigma: v_{l}=v_{Y} / \sqrt{1-2 \sigma^{2} /(1-\sigma)}$

[24] The dimensions of the reeds have been $h=160 \mu \mathrm{m}, l=3.5$ and $11 \mathrm{~mm}$ (Suprasil W), $h=160 \mu \mathrm{m}$, $l \approx 5 \mathrm{~mm}$ (cover glass) and $h \approx 50 \mu \mathrm{m}, l=4 \mathrm{~mm}\left(\mathrm{Pd}_{30} \mathrm{Zr}_{70}\right)$ leading to frequencies of the ground mode of 11.4 and $1.2 \mathrm{kHz}$, respectively (Suprasil W), $5.1 \mathrm{kHz}$ (cover glass) and $3.1 \mathrm{kHz}$ $\left(\mathrm{Pd}_{30} \mathrm{Zr}_{70}\right)$. All samples had a width of approximately $2 \mathrm{~mm}$

[25] G. Frossati, R. Maynard, R. Rammal, D. Thoulouze, J. de Phys. Lettres 38 (1977) L 153

[26] L.D. Landau, Course of Theoretical Physics Vol. I, Mechanics, Pergamon Press, Oxford 1982

[27] The strain in a vibrating reed experiment varies both along and across the sample. The maximum strain amplitudes occurring at the clamping position can be estimated only roughly with an uncertainty of a factor of three. Nevertheless, the numbers give coarsely the magnitude of the deformations affecting a considerable part of the reed

[28] J.E. Graebner, B. Golding, R. J. Schutz, F.S.L. Hsu, H.S. Chen, Phys. Rev. B39 (1977) 1480

[29] H. Neckel, P. Esquinazi, G. Weiss, S. Hunklinger, Solid State Comm. 57 (1986) 151

[30] P. Esquinazi, H.M. Ritter, H. Neckel, G. Weiss, S. Hunklinger, Z. Phys. B64 (1986) 81

[31] Yu. Kagan, N. V. Prokof'ev, JETP Lett. 45 (1987) 116; Yu. Kagan, N.V. Prokof'ev, Solid State Comm. 65 (1988) 1385

[32] J. Stockburger, U. Weiss, R. Goerlich, Z. Phys. B84 (1991) 457

[33] K. Dransfeld, Verh. Dtsch. Phys. Ges., Vol. 15 (3) (1980) 400 
[34] Yu.M. Galperin, V.L. Gurevich, D.A. Parshin, J. Physique Lett. 45 (1984) L747

[35] B.D. Laikhtman, Phys. Rev. B29 (1984) 3601

[36] B. Golding, J.E. Graebner, A. B. Kane, J.L. Black, Phys. Rev. Lett. 41 (1978) 1487

[37] P. Doussineau, C. Frenois, R. G. Leisure, A. Levelut, J. Y. Prieur, J. Physique 41 (1980) 1193

[38] S. Hunklinger, Phonon Scattering in Condensed Matter, Solid-State Sciences 51, eds. W. Eisenmenger, K. Lassmann, S. Döttinger, Springer, Berlin Heidelberg New York 1984, 378

[39] J.E. Van Cleve, PhD thesis, Cornell University (1991) (unpublished)

[40] P. Neu, A. Wurger, appears in Z. Phys. B

[41] See for a review: U. Weiss, Quantum Dissipative Dynamics, Series in Modern Condensed Matter Physics, Vol. 2, World Scientific, Singapore 1993

[42] G. Weiss, Materials Science and Engineering A 133 (1991) 45

[43] D.A. Parshin, Z. Phys. B91 (1993) 367 